\title{
Proximal iliotibial band syndrome - a recently described disease
}

\begin{abstract}
Hip joint overload injuries usually occur in sports practitioners, and presently, because of advances in diagnostic imaging tests, principally MRI, they are often diagnosed. Patients generally complain of pain at the proximal insertion of ITB (iliac tubercle: located posteriorly to the anterior superior iliac spine), exacerbated by Ober's maneuver and local palpation.

MRI has an essential role in the diagnosis since the clinical picture can be confused with several diseases - intra and extra-articular circumstances of the hip. At this point, however, it should be noted that the examination should be requested not only for the investigation of the hip but rather to encompass the entire iliac, otherwise, the proximal portion of the ITB will not be properly analyzed. We intend to present a case of proximal iliotibial band syndrome, a recently described disease, still little known by physicians.
\end{abstract}

Keywords: Iliotibial Band Syndrome/diagnosis, Fascia/injuries, Muscle, Skeletal/ injuries, Magnetic Resonance Imaging
Volume 10 Issue 2 - 2018

\author{
Márcio Luís Duarte,' André de Queiroz \\ Pereira da Silva, ${ }^{2}$ Bruno Fernandes Barros \\ Brehme de Abreu,' José Luiz Masson de \\ Almeida Prado,' Marcelo de Queiroz Pereira \\ da Silva \\ 'Radiology,WEBIMAGEM, Brazil \\ ${ }^{2}$ Radiology, CADI Diagnostico, Brazil
}

Correspondence: Marcio Luis Duarte,WEBIMAGEM, Avenida Marques de Sao Vicente 446, Sao Paulo, Sao Paulo, Brazil, Email marcioluisduarte@gmail.com

Received: November 22, 2017 | Published: January 17, 2018
Abbreviations: MRI, Magnetic Resonance Imaging, ITB, Iliotibial Band

\section{Introduction}

Hip joint overload injuries usually occur in sports practitioners, and presently, because of advances in diagnostic imaging tests, principally MRI, they are often diagnosed. ${ }^{1,2}$ Sher et al. ${ }^{1}$ studied group patients referring pain and edema in the region of the iliac tubercle and demonstrated that T2-weighted MRI shows an increase in the iliotibial band (ITB) signal along the lower border of the iliac tubercle.

The fascia lata gets thicker in its insertion in the lower border of the iliac tubercle with the formation of ITB. ${ }^{1,3}$ ITB is a longitudinal orientation fibrous structure that acquires a contribution from gluteus maximus tendon and tensor of the fascia lata in the greater trochanter. ${ }^{1-3}$ ITB inserts into Gerdy's tubercle in the distal region. ${ }^{1,3}$

ITB is unique to humans. It is the most likely part of the adaptive complex associated with vertical posture and bipedal locomotion. ${ }^{2}$ The ITB consists essentially of an excessive use of enthesopathy in its origin in the iliac tubercle. ${ }^{4}$ The ITB is believed to assist the gluteal abductors in maintaining the pelvic position in the coronal plane, providing contralateral (abduction) resistance to pelvic flaccidity or droping of the unsupported limb. ${ }^{2}$

Our goal is to communicate a case of proximal iliotibial band syndrome because it is a recently reported disease that many radiologists are unaware of and consequently do not describe in their reports, delaying the appropriate treatment of the patient.

\section{Case presentation}

37-year-old woman, obese, with pain in the right hip, close to the iliac spine for 06 months, worsening at night. Works as a clerk and walks about 6 kilometers daily. Deny other traumas and physical activity. At physical examination, the patient reported pain on palpation of the iliac tubercle on the right and positive test on Ober's maneuver. Ely test and contracture test of the posterior thigh muscles negative. No edema or bruising at the site of pain inspection. MRI demonstrated swelling around the right anterior iliac spine and sign change of the iliotibial band inserting, compatible with proximal iliotibial band syndrome (Figures $1 \& 2$ ). With the diagnosis, the patient was advised to perform rest, treatment with anti-inflammatory and physical therapy - shock wave therapy and muscle strengthening, in addition to diet for weight loss.
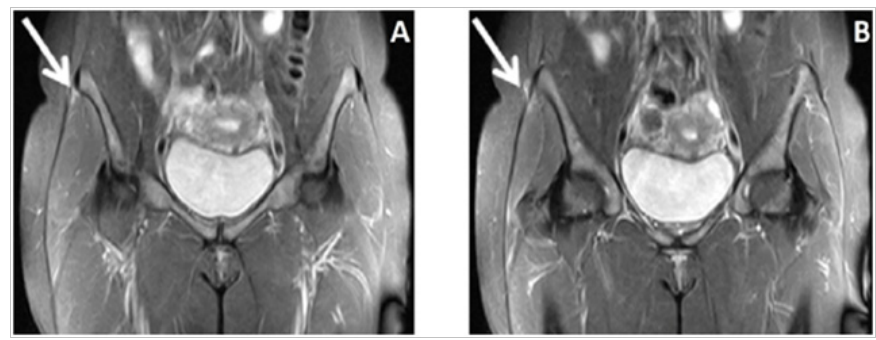

Figure I MRI T2 FAT SAT in coronal section in A and B demonstrating edema around the anterior superior iliac spine and high signal in the right ITB, compatible with the proximal iliotibial tract syndrome (white arrow).

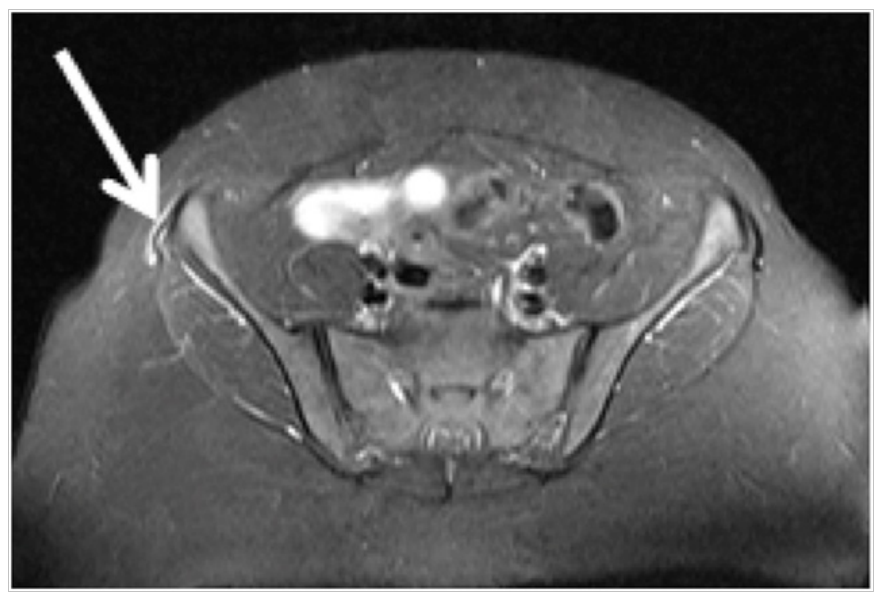

Figure 2 MRI T2 FAT SAT in axial section demonstrates edema around the anterior superior iliac spine and high signal in the right ITB, compatible with the proximal iliotibial tract syndrome (white arrow). 


\section{Discussion}

Symptomatology is generally related to the friction syndrome of ITB distal insertion, which presents with pain in the lateral region of the knee related to ITB friction in the lateral femoral condyle. It usually occurs in long-distance runners, cyclists, and football players and represents the most common cause of lateral knee pain in runners. ${ }^{1}$

The involvement of the proximal region of the ITB is barely discussed in the current literature. The vast majority of reported cases involve female patients - especially runners - or obese elderly women. According to biomechanical studies, a greater relationship between pelvis width and femur length, which occurs in women, generates a greater adductor movement of the hip and generates overload in the lateral muscles in order to maintain pelvic balance. While running, women have a greater activity of the adductor muscles and a greater degree of internal rotation of the hip, which also leads to ITB overload. ${ }^{1,2}$

Patients generally complain of pain at the proximal insertion of ITB (iliac tubercle: located posteriorly to the anterior superior iliac spine), exacerbated by Ober's maneuver and local palpation. ${ }^{1}$ MRI has an essential role in the diagnosis since the clinical picture can be confused with several diseases - intra and extra-articular circumstances of the hip. At this point, however, it should be noted that the examination should be requested not only for the investigation of the hip but rather to encompass the entire iliac, otherwise, the proximal portion of the ITB will not be properly analyzed. ${ }^{1}$

MRI demonstrates signal increase in T2 STIR sequences around the insertion of the iliotibial band in the iliac tubercle and may also show partial thickening and / or partial rupture of its proximal insertion.4 Bone edema of the iliac tubercle is uncommon. ${ }^{4}$ All patients reported in the literature responded to resting and physiotherapeutic treatment for stretching and muscle strengthening of IBT, in addition to antiinflammatories. ${ }^{1,4}$

\section{Acknowledgements}

Not applicable.

\section{Conflicts of interest}

The authors declare that there is no conflict of interests regarding the publication of this paper.

\section{Patient consent}

The written informed consent of the patient was obtained, for the publication of her case.

\section{References}

1. Falótico GG, Yanagishita CMA, Wever AAN, et al. Proximal iliotibial band syndrome: case report. Rev bras ortop. 2013;48(4):374-376.

2. Sher I, Umans H, Downie SA, et al. Proximal iliotibial band syndrome: what is it and where is it? Skeletal Radiol. 2011;40(12):1553-1556.

3. Huang BK, Campos JC, Michael Peschka PG, et al. Injury of the gluteal aponeurotic fascia and proximal iliotibial band: anatomy, pathologic conditions, and MR imaging. Radiographics. 2013;33(5):1437-1452.

4. Flato R, Passanante GJ, Skalski MR, et al. The iliotibial tract: imaging, anatomy, injuries, and other pathology. Skeletal Radiol. 2017;46(5):605-622. 